\title{
Spacecraft conceptual design for the 8-meter Advanced Technology Large Aperture Space Telescope (ATLAST)
}

\author{
Randall C. Hopkins*a, Peter Capizzo ${ }^{\mathrm{b}}$, Sharon Fincher ${ }^{\mathrm{a}}$, Linda S. Hornsby ${ }^{\mathrm{c}}$, David Jones ${ }^{\mathrm{a}}$, \\ Gary Mosier ${ }^{\mathrm{d}}$, H. Philip Stahl ${ }^{\mathrm{a}}$, Dan Thomas ${ }^{\mathrm{a}}$, Kevin S. Thompson ${ }^{\mathrm{c}}$ \\ ${ }^{a}$ NASA Marshall Space Flight Center, AL, USA 35812; \\ ${ }^{\mathrm{b}}$ Raytheon, Marshall Space Flight Center, AL, USA 35812; \\ ${ }^{c} J a c o b s$ ESTS Group, Marshall Space Flight Center, AL, USA 35812; \\ dNASA Goddard Space Flight Center, MD, USA 20771;
}

\begin{abstract}
The Advanced Concepts Office at Marshall Space Flight Center completed a brief spacecraft design study for the 8meter monolithic Advanced Technology Large Aperture Space Telescope (ATLAST-8m). This spacecraft concept provides all power, communication, telemetry, avionics, guidance and control, and thermal control for the observatory, and inserts the observatory into a halo orbit about the second Sun-Earth Lagrange point. The multidisciplinary design team created a simple spacecraft design that enables component and science instrument servicing, employs articulating solar panels for help with momentum management, and provides precise pointing control while at the same time fast slewing for the observatory.
\end{abstract}

Keywords: ATLAST, large aperture space telescope, monolithic mirror, astronomy, exo-planets.

\section{INTRODUCTION}

The Advanced Technology Large Aperture Space Telescope (ATLAST) Astrophysics Strategic Mission Concept Study ${ }^{1,2}$ examined several potential architectures to achieve high priority science:

- Is there Life elsewhere in the Galaxy: characterization of exo-planets

- Star Formation and Evolution: resolved stellar populations

- Galaxy Formation and Evolution: super-massive black hole evolution

- Formation of Structure in the Universe: dark matter kinematics

- Planetary Science: origin and nature of objects in the outer solar system

One of the three design concepts examined was an 8-meter monolithic aperture ATLAST (ATLAST-8m) for which NASA MSFC developed a detailed preliminary point design. ATLAST-8m is a potential next generation flagship UVOIR space observatory (wavelength coverage: $110 \mathrm{~nm}-2400 \mathrm{~nm}$ ). By virtue of its $\sim 15$ milli-arcsec (mas) angular resolution at $\sim 500 \mathrm{~nm}$ coupled with its ultra high sensitivity, superb stability and low sky background, ATLAST-8m will achieve major breakthroughs in astrophysics by enabling fundamentally new observations. ATLAST-8m has the optical performance required to detect the potentially rare occurrence of bio-signatures in the spectra of terrestrial exo-planets, to reveal the underlying physics that drives star formation, and to trace the complex interactions between dark matter, galaxies, and the intergalactic medium. ${ }^{1,2}$ ATLAST- $8 \mathrm{~m}$ will be able to observe the habitable zone of 145 long-lived stars (spectral type F,G,K) up to $\sim 25$ parsecs. ATLAST-8m will be able to study star formation histories in the local universe by measuring accurate colors and luminosities of stars in over 100 galaxies, of which over ten are giant spirals and one is a giant elliptical. And, ATLAST-8m will enable the study of galaxy and large structure formation by directly measuring key properties (both for dark and visible matter) of structures and mapping their evolution in time.

*Randall.Hopkins@nasa.gov; phone 1 256 544-4977; fax 1256 544-0242; www.nasa.gov/centers/marshall/advanced_concepts 
ATLAST-8m was specifically designed to take advantage of the mass and volume capacities of a potential heavy lift launch vehicle such as the Ares $\mathrm{V}^{3}$ to achieve exceptional optical performance and long-term stability. Performance is primarily achieved via volume: volume allows for an $8-\mathrm{m}$ diameter monolithic aperture telescope. Stability is primarily achieved via mass: structural mass to achieve very stable long-duration pointing; and thermal mass to achieve longduration optical wavefront stability.

While the scientific benefit of space-based telescopes is well established, designing spacecraft to point and control large monolithic telescopes is challenging. In particular, the 8-meter monolithic Advanced Technology Large Aperture Space Telescope, with its 19 metric ton primary mirror and more than 50 metric ton total observatory mass, poses many pressing design constraints on a spacecraft. Figure 1 shows the ATLAST-8m observatory in its deployed configuration and its major structural and optical elements. The primary mirror is located at the rear of the optical tube assembly (OTA), with the center of gravity very close to this point. The spacecraft is attached to the rear of the OTA as shown. The text below describes a conceptual spacecraft design capable of controlling the 8-meter telescope. The telescope structural design ${ }^{4}$ is described elsewhere.

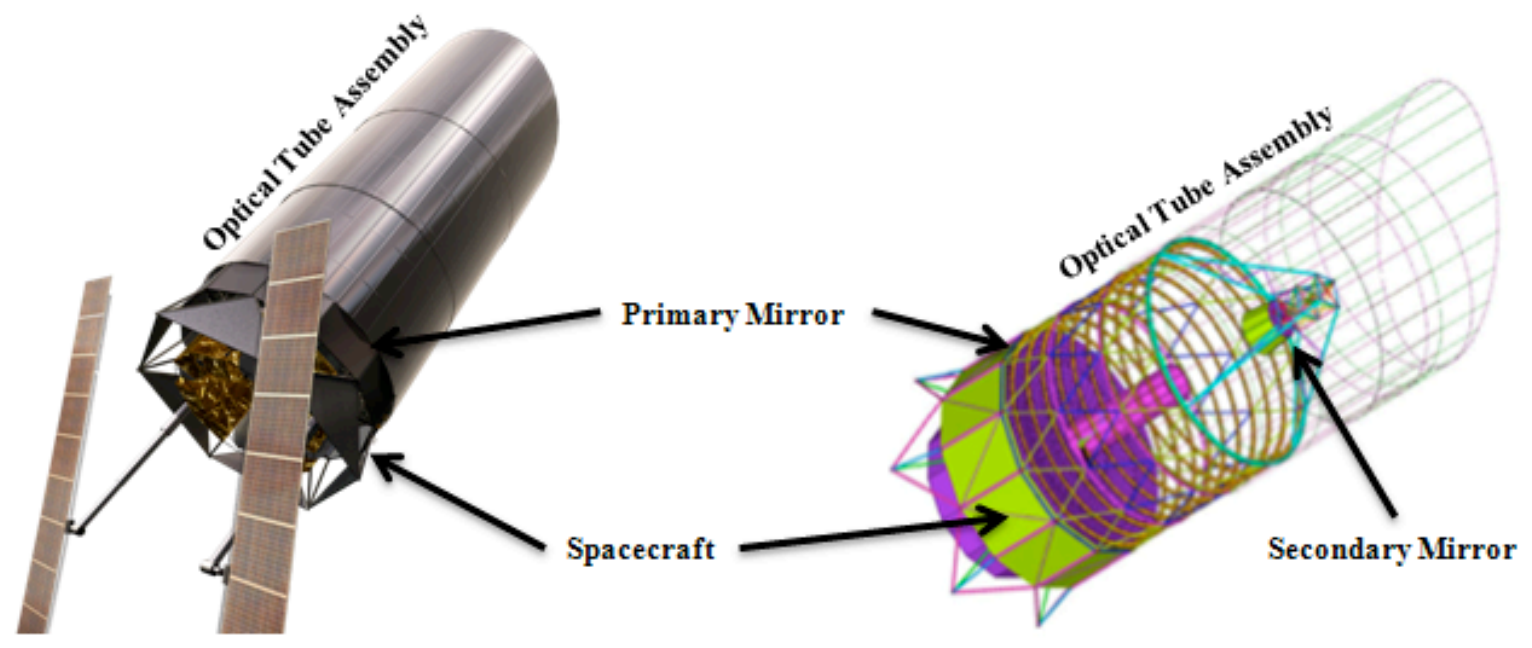

(a)

(b)

Figure 1. Illustration showing the (a) deployed observatory and (b) the major structural and optical elements.

\section{GROUND RULES AND ASSUMPTIONS}

The spacecraft provides all pointing, power, communications, navigation, thermal control, data storage, station keeping and momentum unloading for the observatory, and inserts it into the target halo orbit about the second Sun-Earth Lagrange point (SE-L2). It also executes mid-course correction maneuvers and transfer trajectory insertion error corrections. The spacecraft is designed such that subsystem components are grouped into on-orbit replaceable units (ORUs) to allow efficient removal and replacement during robotic and/or human servicing. Top-level spacecraft requirements are listed in Table 1.

Additional ground rules and assumptions may be listed in the subsystem sections below. Payload for the spacecraft consists of the 8-meter telescope and several science instruments. The science instrument volume is illustrated in Section 4.1 below, with power and thermal requirements described in the appropriate sections. More information on the science instruments can be found in Postman's paper ${ }^{1}$ and on the ATLAST website. ${ }^{2}$ 
Table 1. Top-level ATLAST 8-meter requirements.

\begin{tabular}{|l|l|}
\hline Requirement & Value \\
\hline Orbit & Halo orbit about Sun-Earth L2 \\
\hline Sun avoidance angle & 60 degrees \\
\hline Fast Slew Requirement & 60 degrees in 90 minutes required; 40 minutes desired \\
\hline Pointing Accuracy & 1.6 milli-arc seconds \\
\hline Inertial Pointing & 4500 minutes required; 9000 minutes desired \\
\hline Roll Angle and Rate & \pm 30 degrees in 60 minutes required; 30 minutes desired \\
\hline Primary Mirror Temperature and Range & $280 \mathrm{~K} \pm 4 \mathrm{~K}$ \\
\hline Lifetime for Spacecraft & $\begin{array}{l}5 \text { years for serviceable components unless otherwise specified; } \\
20 \text { years for propulsion system. }\end{array}$ \\
\hline
\end{tabular}

\section{MISSION ANALYSIS}

The target orbit for the ATLAST 8-meter observatory is a halo orbit about SE-L2, approximately 1.5 million kilometers from Earth. This orbit provides a stable thermal environment for the massive primary mirror, and is largely free of interference from Earth, the moon, and the sun, provided the 60-degree sun avoidance angle is observed. The chosen transfer trajectory and final orbit are similar to that of the James Webb Space Telescope. Major mission design parameters are listed in Table 2.

At the time of this study, the Ares $\mathrm{V}$ heavy lift vehicle was still in the preliminary design phase, but the chosen configuration was capable of placing $65000 \mathrm{~kg}$ onto the $\mathrm{L} 2$ transfer trajectory with a $\mathrm{C} 3$ of $-0.7 \mathrm{~km}^{2} / \mathrm{s}^{2}$. Even though the proposed NASA budget calls for canceling the Constellation Program and thus the Ares V, the new heavy lift vehicle design study at Marshall Space Flight Center is considering launch vehicles with volume and mass capabilities comparable to the Ares V. Therefore, the possibility of launching a large monolithic observatory like the ATLAST 8meter remains.

Table 2. Major mission design parameters.

\begin{tabular}{|l|c|c|}
\hline Parameter & Value & Units \\
\hline Launch Vehicle and Payload Mass & Ares V (51.01.48); 65000 & $\mathrm{kg}$ \\
\hline Mission Lifetime & 60 design, 240 consumables & $\mathrm{mos}$ \\
\hline Transfer Trajectory C3 & -0.7 & $\mathrm{~km} / \mathrm{s}^{2}$ \\
\hline Correction after Transfer Trajectory Insertion & 52 & $\mathrm{~m} / \mathrm{s}$ \\
\hline Midcourse Correction & 10 & $\mathrm{~m} / \mathrm{s}$ \\
\hline SE-L2 Stationkeeping for 20 years & 80 & $\mathrm{~kg}$ \\
\hline Momentum Unloading for 20 years & 21.2 & $\mathrm{~kg}$ \\
\hline
\end{tabular}

\section{SPACECRAFT DESIGN}

\subsection{Integrated Design and Mass Budget}

The ATLAST-8m spacecraft is an integration of subsystems required to provide support for the ATLAST-8m telescope and instrument. The spacecraft subsystems provide all pointing, navigation, communication, thermal control, power, data storage and transmission, station keeping and momentum dumping. A modular spacecraft design enables some subsystems to be serviced or replaced on-orbit. The spacecraft is designed with the assumption of servicing at regular intervals, with most serviceable subsystems designed for a 5 year lifetime and the remaining serviceable subsystems for a 20 year lifetime, unless otherwise specified. Figure 2 below shows a three-dimensional cross-sectional view of the spacecraft envelope. The spacecraft is positioned around the instrument volume and within the Ares $\mathrm{V}$ launch vehicle payload attachment fixture (PAF).

The challenge of the spacecraft integrated design is to package all the required subsystems in the space allocation while allowing for required servicing access. Also, minimizing deployment mechanisms to increase reliability is a primary consideration. The resulting integrated spacecraft design incorporates a modular approach for packaging the serviceable 
components. The payload adapter and telescope support structures are utilized for mounting the spacecraft radiators. Also, there are two docking locations for the service vehicle. The passive trunnions for the automated rendezvous and docking (AR\&D) mechanisms are located 180 degrees apart and also utilize the telescope support structure for mounting. A preliminary concept of the integrated spacecraft design is shown in Figure 3.

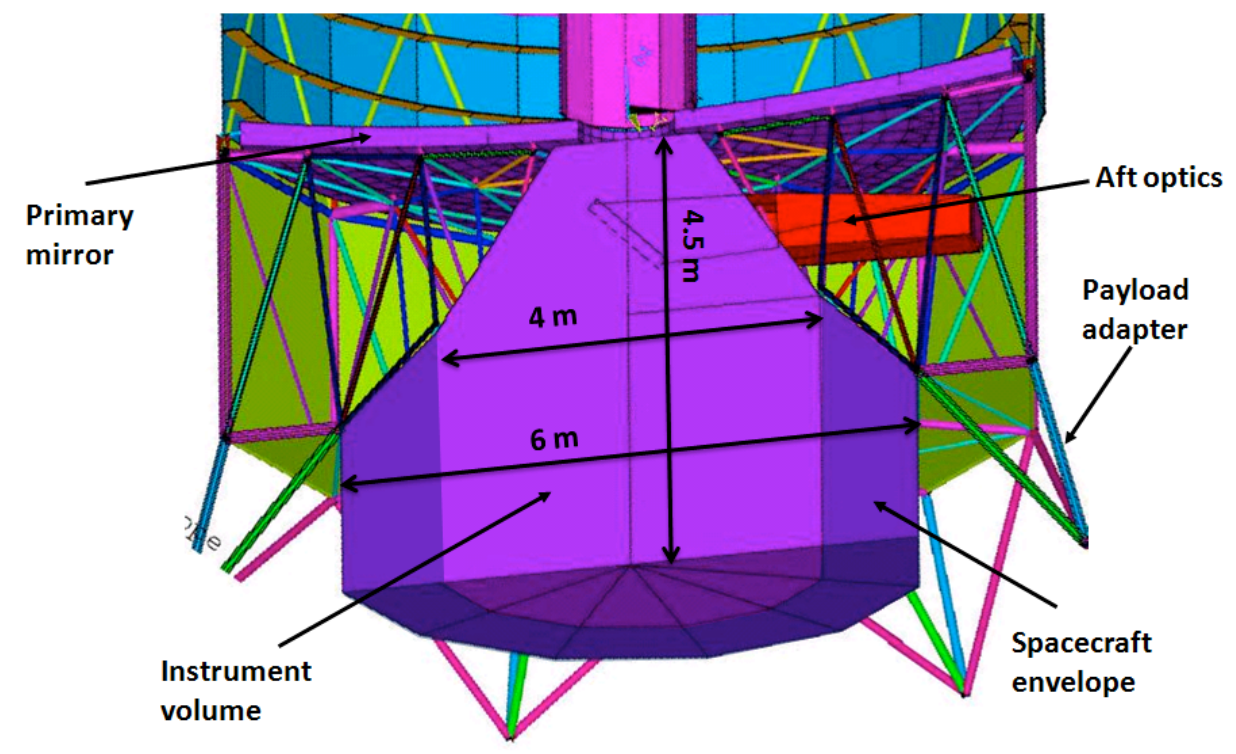

Figure 2. Cutaway showing the spacecraft envelope.

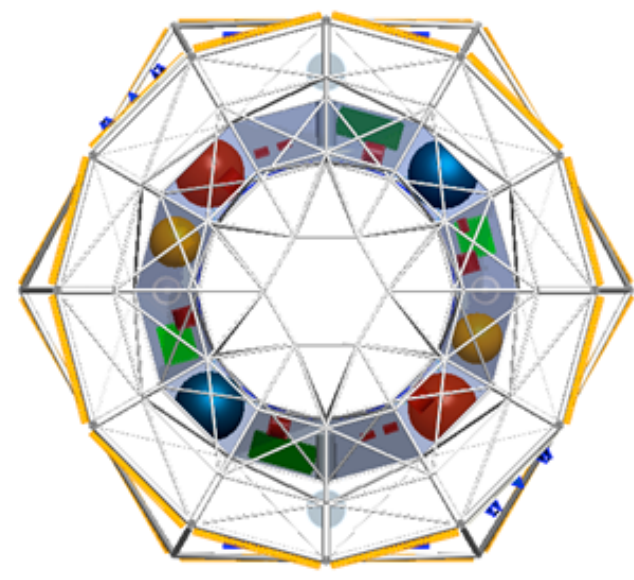

Top View

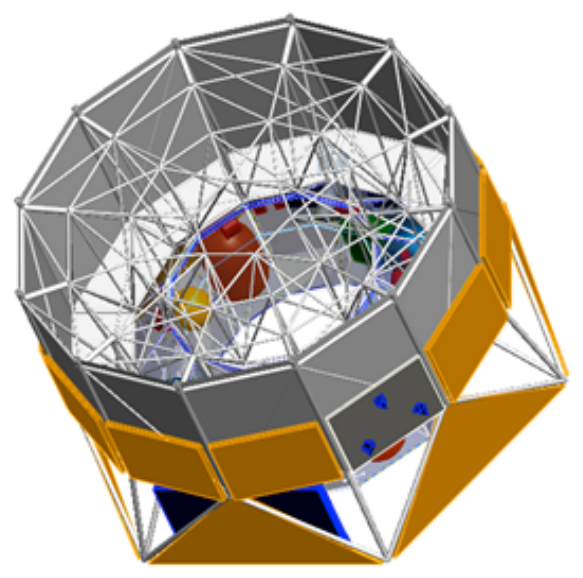

Isometric View

Figure 3. Spacecraft configuration views showing how the subsystem components are arranged in a donut pattern around the science instrument volume.

The ATLAST integrated spacecraft and the telescope packaged in the ARES V 10-m payload fairing are shown in Figure 4. The ARES V ogive payload fairing provides adequate volume for packaging the spacecraft stowed configuration integrated with the stowed telescope. The ogive shroud has an 8.8 meter diameter dynamic envelope.

The only ATLAST spacecraft mechanisms requiring development are for the solar arrays, which deploy aft of the spacecraft as shown in Figure 1(a). (See also Section 6 below.) The solar array deployment mechanisms must allow the 
arrays to gimbal and retract as required. For example, the solar arrays will need to be retracted during the docking of the service vehicle to avoid interference and also prevent contamination of the arrays by the vehicle propulsion systems.

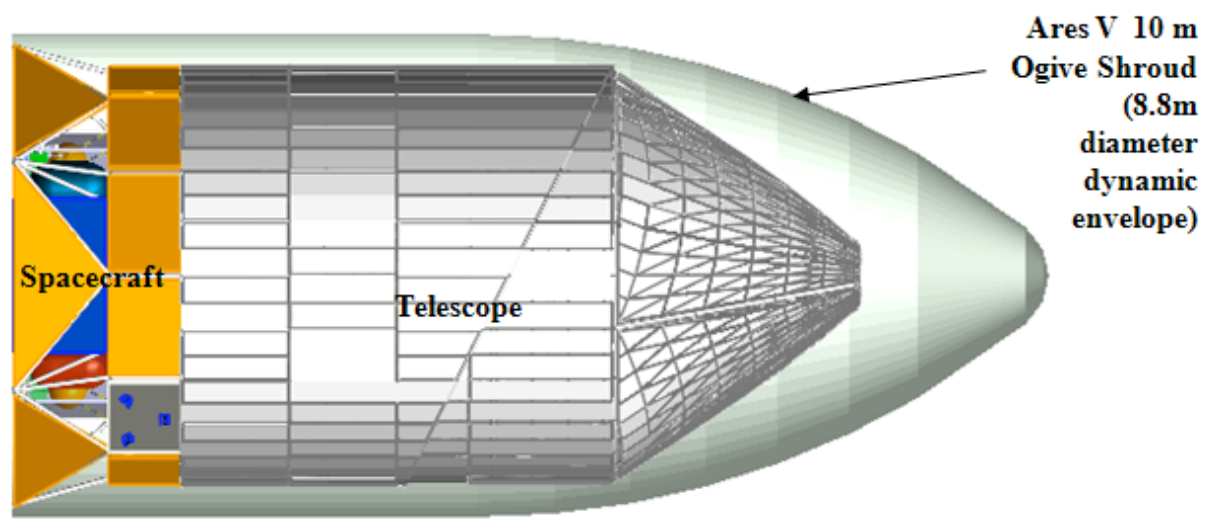

Figure 4. ATLAST 8-meter observatory stowed within the Ares V ogive shroud.

Table 3. Top-level mass statement for the ATLAST 8-meter observatory.

\begin{tabular}{|c|c|c|c|c|}
\hline Element & mass total & $\begin{array}{l}\text { growth } \\
\text { margin } \\
(\%)\end{array}$ & growth & $\begin{array}{l}\text { Mass with } \\
\text { growth } \\
\text { (kg) }\end{array}$ \\
\hline Optical Telescope Assembly (OTA) & 33,908 & $30.00 \%$ & 10,172 & 44,080 \\
\hline Science Instruments & 1,789 & $30.00 \%$ & 537 & 2,326 \\
\hline Spacecraft & 4,157 & & 1,247 & 5,404 \\
\hline Propulsion System & 401 & & 120 & 521 \\
\hline Electrical Power System & 1,104 & & 331 & 1,435 \\
\hline Thermal Control System & 554 & & 166 & 720 \\
\hline Attitude Control System & 499 & & 149.7 & 649 \\
\hline Communications System & 114 & & 34.2 & 148.2 \\
\hline Command and Data Handling System & 140 & & 42 & 182 \\
\hline Structures and Mechanisms System & 1,345 & & 404 & 1,749 \\
\hline Observatory Dry Mass & 39,854 & & 11,956 & 51,810 \\
\hline Propellant & 4,666 & & $\mathbf{0}$ & 4,666 \\
\hline MMH & 1752 & $0.00 \%$ & 0 & 1,752 \\
\hline $\mathrm{N} 2 \mathrm{O} 4$ & 2891 & $0.00 \%$ & 0 & 2,891 \\
\hline Pressurant (Helium) & 23 & $0.00 \%$ & 0 & 23 \\
\hline TOTAL OBSERVATORY MASS & 44,520 & & 11,956 & 56,476 \\
\hline
\end{tabular}

\subsection{Propulsion System (PS)}

The propulsion system is responsible for correcting the launch vehicle orbital insertion errors and performs any midcourse correction maneuvers required during the transfer to the Sun-Earth L2 point. While at the SE-L2, the PS is also used for station-keeping and momentum unloading of the reaction wheels. Due to the large $\Delta \mathrm{V}$ requirements, as well as the long mission duration, a storable bipropellant system (pressure-fed) is chosen with liquid MMH and N2O4 as the fuel and oxidizer, respectively. High-pressure gaseous helium is used to maintain desired pressures in the propellant tanks, which ensures nominal engine performance during the entire mission span.

The PS includes two $900 \mathrm{lbf}$ thrusters and four thruster pods, each containing two $25 \mathrm{lbf}$ and two $5 \mathrm{lbf}$ engines. The 900 lbf engines perform the large L2 orbit insertion burn (if required, depending on the transfer trajectory), whereas the 25 $\mathrm{lbf}$ engines are responsible for the maneuvers that are too small for the large engines but too large for the smaller $5 \mathrm{lbf}$ 
thrusters (these events include the launch error clean-up, midcourse corrections and orbit station-keeping). Finally, the $5 \mathrm{lbf}$ engines are used to de-saturate the momentum wheels that maintain the spacecraft's attitude.

The system design consists of mostly flight-proven components. The propellant load of $4643 \mathrm{~kg}(1752 \mathrm{~kg}$ of fuel and $2891 \mathrm{~kg}$ of oxidizer) is stored in two fuel and two oxidizer tanks. A single design is used for all four tanks: ATK-803951 . The $23 \mathrm{~kg}$ of gaseous helium is stored in 6 high-pressure tanks. This allows the use of the ATK-80436-1 tank. Off-theshelf components are also used for valves, filters, regulators, and instrumentation. These items, along with the other system components, are listed below in Table 4 . The total dry mass of the propulsion system, without a growth margin, is $401 \mathrm{~kg}$. Applying 30\% raises the mass to $521 \mathrm{~kg}$.

Table 4. Propulsion system mass summary.

\begin{tabular}{|c|c|c|c|c|c|c|}
\hline Item & QTY & $\begin{array}{c}\text { Unit Mass } \\
(\mathrm{kg})\end{array}$ & $\begin{array}{c}\text { Basic Mass } \\
(\mathrm{kg})\end{array}$ & $\begin{array}{c}\text { Margin } \\
(\%)\end{array}$ & $\begin{array}{c}\text { Predicted Mass } \\
(\mathrm{kg})\end{array}$ & Comments \\
\hline Pressurant Fill/Drain Valve & 12 & 0.21 & 2.52 & 30 & 3.28 & Moog (50E889) \\
\hline Pressurant Filter & 4 & 0.25 & 1.00 & 30 & 1.30 & Vacco (F1D10636-01) \\
\hline Pressure Regulator & 4 & 2.04 & 8.16 & 30 & 10.61 & Vacco (83735-1) \\
\hline Pressure Transducer & 28 & 0.23 & 6.44 & 30 & 8.37 & Messenger Hardware \\
\hline Check Valves & 8 & 0.27 & 2.16 & 30 & 2.81 & Messenger Hardware \\
\hline Burst Disk & 4 & 0.10 & 0.40 & 30 & 0.52 & STS OMS \\
\hline Relieve Valve & 4 & 2.31 & 9.24 & 30 & 12.01 & STS OMS \\
\hline Propellant Fill and Drain Valve & 4 & 0.21 & 0.84 & 30 & 1.09 & Moog (50E889) \\
\hline Propellant Filter & 4 & 0.91 & 3.64 & 30 & 4.73 & Vacco (F1D10648-01) \\
\hline Latch Valve & 9 & 0.80 & 7.20 & 30 & 9.36 & Vacco (V1E10560-01) \\
\hline Ground Checkout Valve & 4 & 0.07 & 0.28 & 30 & 0.36 & Messenger Hardware \\
\hline Pressurant Tank (4967 in $\left.{ }^{3}\right)$ & 6 & 12.70 & 76.19 & 30 & 99.04 & ATK (80436-1) \\
\hline Propellant Tank (78647 in $\left.{ }^{3}\right)$ & 4 & 42.28 & 169.14 & 30 & 219.88 & ATK (80395-1) \\
\hline MPS Thruster (900 lbf) & 2 & 6.80 & 13.60 & 30 & 17.68 & Aerojet R-40B \\
\hline Large RCS Thruster $\left(25 \mathrm{lbf}^{2}\right)$ & 8 & 2.00 & 16.00 & 30 & 20.80 & Aerojet R-1E \\
\hline Small RCS Thruster $\left(5 \mathrm{lbf}^{2}\right)$ & 8 & 0.45 & 3.60 & 30 & 4.68 & Aerojet R-6D \\
\hline Lines and fittings & 1 & 38.40 & 38.40 & 30 & 49.92 & Estimate \\
\hline Structural Mounts & 1 & 42.20 & 42.20 & 30 & 54.86 & Estimate \\
\hline
\end{tabular}

\subsection{Avionics, Power, and GN\&C}

Attitude Control System (ACS). The main components of the ACS include the Fine Guidance Sensor integrated into the science payload; a coarse pointing system on the SC that includes gyros, star trackers, reaction wheel assemblies (RWA), and sun sensors; a propulsion system that provides attitude control (in certain situations) and momentum unloading; active vibration isolation system between the spacecraft and the observatory; and software resident on the SC Bus C\&DH system that runs the ACS algorithms, which enable the ACS components to work together as intended.

The spacecraft ACS components are generally 1-fault tolerant for a 5 year life with regular ORU servicing planned. Some critical components such as star trackers are designed for 10 years. Since the telescope avionics systems are not to be normally serviced, they are 2-fault tolerant for a 20 year life. Because of the mass, size, and pointing time required for the observatory, the reaction wheel assembly (RWA) design is challenging. Using up to 12 MWI-100/100 Teldix reaction wheels (700 Nms momentum storage) still could not meet the $4500 \mathrm{sec}$ continuous observation time. To meet the observation time, a solar pressure balancing strategy using solar arrays is employed. The solar arrays are mounted on extendable booms that are maneuvered to balance solar pressure on the optical tube assembly. Assuming restrictions in solar array movement during observations and resulting balancing errors, a curve of boom length versus pointing times was established. With as much as $27.5 \%$ margin on the SA optimal boom length, the 4500 minute observation time can still be achieved. With only a $13 \%$ margin, the 9000 minute observation goal can be achieved. By using the isolation 
systems described below and allowing unrestricted solar array movement during observations, unlimited observation times are feasible, exceeding mission goals.

A 12 wheel RWA is probably not practical and is used as a mass placeholder. Development of larger reaction wheels or a Control Moment Gyro (CMG) system will be necessary. However, a CMG system is presently flying on the ISS and need only be scaled for the ATLAST mission. Using CMGs would also allow the desired slew rate of 60 degrees in 40 minutes to be easily achieved because of the much greater torque capability over reaction wheels.

The active vibration isolation system has two roles. First, it isolates the science payload from SC bus disturbances, such as vibrations from the RWA. Second, it provides fine pointing control for the science payload, which requires a pointing stability of 1.6 mas. During science observations, the OTA bore-sight is pointed to within a few arcsec of the desired target using the RWA. The active isolation system then engages to minimize the apparent motion of the guide star centroid for the duration of that science exposure. When sensors indicate that the voice coil actuators (VCA) are approaching their travel limits, the ACS software commands the RWAs to produce a small, gradual change in the orientation of the SC bus. This causes the VCAs to move back near the center of their travel as they adjust to minimize the apparent movement of the guide star centroid. While slewing, the control bandwidth is increased so that the buspayload connection is stiffened. This feedback loop between FGS, active isolation system, and RWA continues until the end of the science observation.

Except for active isolation and reaction wheels/CMG, the ACS components on the SC bus are all TRL 8. Active isolation is considered to have a TRL of 4 based on the Lockheed Martin demonstration of their Disturbance Free Payload system. ${ }^{5}$ It should be noted that Northrop Grumman, CSA and others have demonstrated similar capabilities. The reaction wheel or CMG systems to be scaled are TRL 7.

Command and Data Handling System (C\&DHS). The C\&DH system is straightforward and can be implemented with current technology. Its architecture supports the ORU modularity of the SC Bus. The flight computer and data recorder contain internal redundancy in accommodation of the ORU strategy. The flight computer communicates with the science instrument processors to collect and transfer data to the data recorder and transmitters at a rate of $600 \mathrm{Mbps}$. The flight computer is also responsible for all spacecraft operations and functionality, such as solar array sun pointing and pressure balancing, reaction wheel pointing control, momentum dumping operations, controlling the isolation subsystem, and thermal management of the primary mirror and spacecraft. The C\&DH system also consist of electronic controllers for the secondary and tertiary mirrors, along with remote data acquisition units for collecting spacecraft health and status information. All C\&DH system components are at least TRL 7.

Communications System (CS). The communications system consists of a Ka-band high gain pointing antenna for a 600 Mbps data downlink, a medium gain system for telemetry and engineering data links, and an S-band system for local communications with a servicing vehicle or for backup capability. Because $600 \mathrm{Mbps}$ communications have not yet been demonstrated in SE-L2 orbit, the CS TRL is 7.

Table 5. Avionics and GN\&C mass and power summary.

\begin{tabular}{|l|l|l|}
\hline Avionics and GN\&C & Mass (kg) & Power (W) \\
\hline Attitude Control System (includes active vibration isolation system) & 499 & 1856 \\
\hline Command and Data Handling System & 140 & 674 \\
\hline Communications System & 114 & 415 \\
\hline Totals & $\mathbf{7 5 3}$ & $\mathbf{2 9 4 5}$ \\
\hline
\end{tabular}

Electrical Power System (EPS). The ATLAST-8m EPS is a robust system, providing the observatory with all required power. Spacecraft power systems are generally 1-fault tolerant for 5 year life, with some components such as batteries and solar arrays designed for 10 years. Since the telescope systems will not be robotically serviceable, the components are generally 2 -fault tolerant for a 20 year life. The power system is designed to provide $3.5 \mathrm{~kW}$ for science instruments and $2 \mathrm{~kW}$ of supplemental power for heating the primary mirror. When instruments are powered down, the EPS system will provide $4 \mathrm{~kW}$ of power for heating the PM. The total observatory EPS provides $11.2 \mathrm{~kW}$ of power with $30 \%$ margin. The SC battery is sized to provide 3 hours of power to the observatory prior to solar array deployment and during servicing operations as necessary. The batteries are $100 \mathrm{Wh} / \mathrm{kg} \mathrm{Li}$-ion. Solar arrays are GaAs $100 \mathrm{~W} / \mathrm{kg}$ triple junction. Total solar array area is $72 \mathrm{~m}^{2}$. The solar arrays are deployed on adjustable booms to counter solar pressure counter on the telescope tube; this balancing system can theoretically nullify all solar torque, providing unlimited observation times. 
The solar arrays are also doubled gimbaled to accommodate all slews and roll maneuvers, while maintaining the solar pressure balance. EPS elements are arranged in ORUs to allow for replacement during servicing missions, and incorporate blind mate connectors. While some solar array mechanisms will need to be space qualified, all EPS components are TRL 8.

Table 6. Mass and power summary for the EPS.

\begin{tabular}{|l|l|l|}
\hline Power Systems & Mass (kg) & Power (W) \\
\hline Power Management and Distribution & 155 & 569 \\
\hline Cabling & 413 & NA \\
\hline Solar Array & 300 & NA \\
\hline Batteries & 236 & NA \\
\hline Heaters & NA & 2382 \\
\hline Science Instruments & NA & $2692^{*}$ \\
\hline Totals & $\mathbf{1 1 0 4}$ & $\mathbf{5 6 4 3}$ \\
\hline
\end{tabular}

*Total science instruments power with $30 \%$ margin is 3500 Watts.

\subsection{Thermal Control System (TCS)}

The passive spacecraft thermal control system consists of radiators, cold plate/heat pipe assemblies, heaters, and multilayer insulation. Figure 5 illustrates a notional thermal control scheme for the spacecraft. The thermal control system is required to maintain allowable operating temperatures for all subsystem and experiment components. The solar flux at an altitude of $1.5 \times 10^{6} \mathrm{~km}$ (consistent with an orbit about L2) is assumed to be $1296 \mathrm{~W} / \mathrm{m}^{2}$.

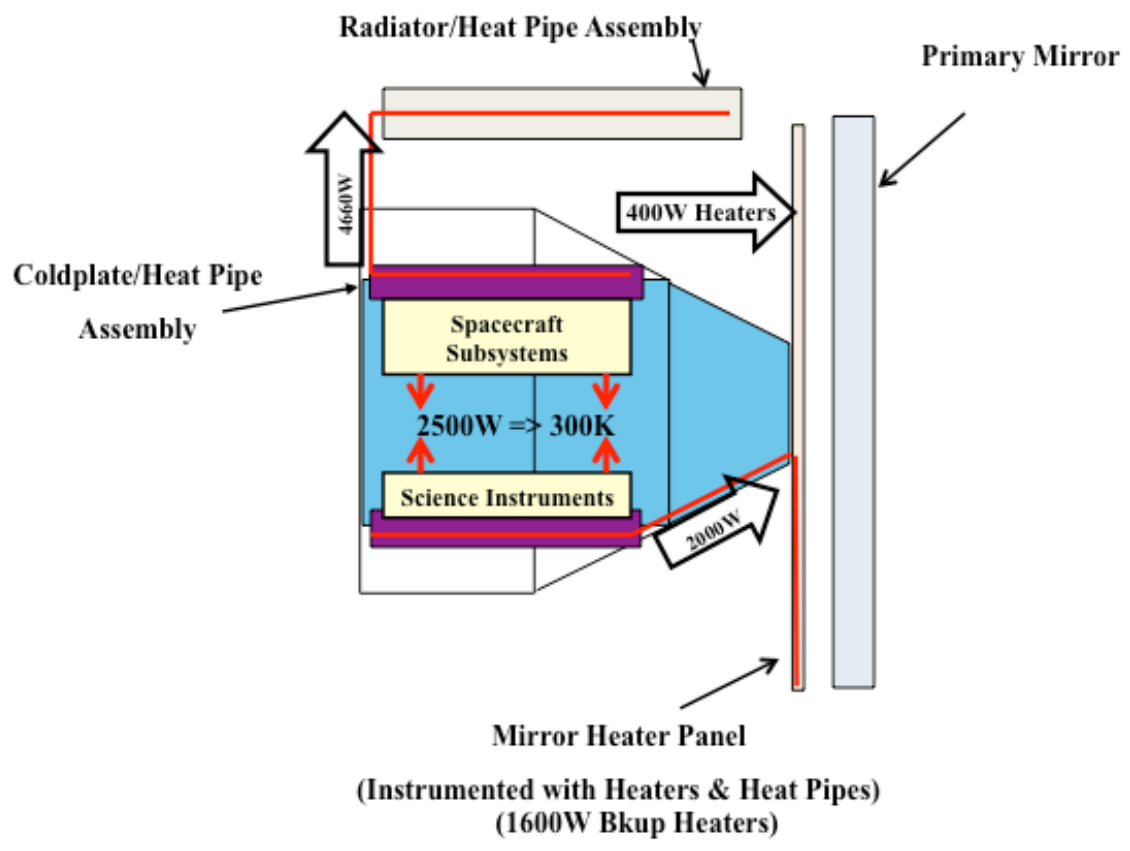

Figure 5. Notional spacecraft thermal control schematic.

Radiators $\left(30 \mathrm{~m}^{2}\right)$ are body mounted around the circumference of the optical telescope assembly and spacecraft and will accommodate 4660 watts of heat dissipation. Spacecraft subsystems components and science instruments are mounted to cold plate/heat pipe assemblies in order to dissipate heat.

The spacecraft will be wrapped in a 50 layer insulation blanket to help minimize the impact of solar heating on the interior (effective emissivity $=0.004$ ). The outer layer of MLI is assumed to be 10 mil silverized Teflon for low solar flux absorbance. The objective is to maintain the on-orbit operational steady state temperature of the spacecraft interior at $300^{\circ} \mathrm{K}\left( \pm 10^{\circ} \mathrm{K}\right)$. This requires 2500 watts of heat, supplied radiatively by science instruments and spacecraft components. The spacecraft propulsion system consists of a conventional pressure-fed bipropellant MMH-NTO system 
with two fuel tanks, two oxidizer tanks, and two pressurant tanks, all located inside the insulated spacecraft compartment.

Table 7 provides details of the power consumption/heat dissipation for the spacecraft subsystems and science instruments. The spacecraft astrionics heat dissipation is assumed equal to the power requirement for the attitude control system (ACS), command and data system (CDS), instrumentation and monitoring (I\&M), \& communications (COMM) systems. The attitude control system will require 1630 watts, the command and data system will require 850 watts, the instrumentation and monitoring will require 806 watts, and the communications system will require 539 watts of power, on average to operate. The power system (batteries and power system enclosure) will dissipate an estimated 3835 watts and the science instruments will dissipate 3500 watts. A total of 11,160 watts must be managed by the spacecraft thermal control system.

Table 7. Power consumption / heat dissipation summary.

\begin{tabular}{|l|l|}
\hline System & \multicolumn{1}{|c|}{$\begin{array}{c}\text { Power/Heat } \\
\text { Dissipation (W) }\end{array}$} \\
\hline Attitude Control System & 1630 \\
\hline Command Data System & 850 \\
\hline Instrumentation \& Monitoring & 806 \\
\hline Communications & 539 \\
\hline Power & 3835 \\
\hline Science Instruments & 3500 \\
\hline Total & $\mathbf{1 1 , 1 6 0}$ \\
\hline
\end{tabular}

The primary mirror is to be maintained at $280 \mathrm{~K},{ }^{6}$ and is thermally managed by wrapping the support structure with a 50 layer MLI blanket. Active heating of the mirror requires up to 4000 watts, to be supplied as 2000 watts of heater power and scavenging up to 2000 watts of excess heat from the instruments via a heat pipe/cold plate assembly. The telescope MLI, heat pipes located behind the mirror, and the heaters and heater structure located behind the mirror are not budgeted with the spacecraft. The preliminary design thermal control mass summary for the ATLAST-8m is shown in Table 8.

Table 8. Thermal control mass summary.

\begin{tabular}{|c|c|}
\hline Element & Mass (kg) \\
\hline MLI & 185.0 \\
\hline Radiators w/heat pipes & 115.0 \\
\hline Equipment cold plates w/heat pipes & 254.0 \\
\hline Total & $\mathbf{9 7 4 . 0}$ \\
\hline
\end{tabular}

All thermal control components are commonly used in spacecraft design and considered to be a high TRL. Heat pipes are always custom designed to specifications and the integration of the heat pipes into the cold plate and primary mirror thermal control design will be a unique application and will require rigorous design and testing.

\subsection{Structures and Mechanisms (SMS)}

During launch, the spacecraft is attached to the rear of the telescope structure, and does not support the observatory nor transfer launch loads to the Ares V launch vehicle (LV). Therefore, the structure is a lightweight and simple design, with aluminum-lithium plate elements and aluminum tubing being the main components. The SC Bus is hard-mounted to the observatory in sixteen locations, and supports all of the internal components through all aspects of the observatory's mission. Figure 6 shows the SC Bus stress plot during launch. The SMS also supports SC Bus servicing by incorporating ORUs that contain most of the active SC Bus components.

After separating from the LV, the SMS deploys the Solar Array (SA) and the steerable High Gain Antenna (HGA). Each of the two solar arrays is connected to a telescoping boom by an articulating mechanism, thus enabling the solar arrays to always be perpendicular to the sun but allowing their distance to the observatory's center of mass to vary. This allows for optimal power from the arrays while utilizing the solar radiation pressure to help balance the solar radiation torque from the telescope's optical tube. 
Except for the solar array deployment mechanisms, all SMS components are considered to be at TRL 6 or higher. For the solar array deployment, the telescoping boom and the articulation mechanism have both flown in space, but both have not been used simultaneously for solar array control. The ATLAST team estimates this component has a TRL of 5. An animation of the solar array deployment is included in Section 6. The S\&MS masses are listed in Table 9.

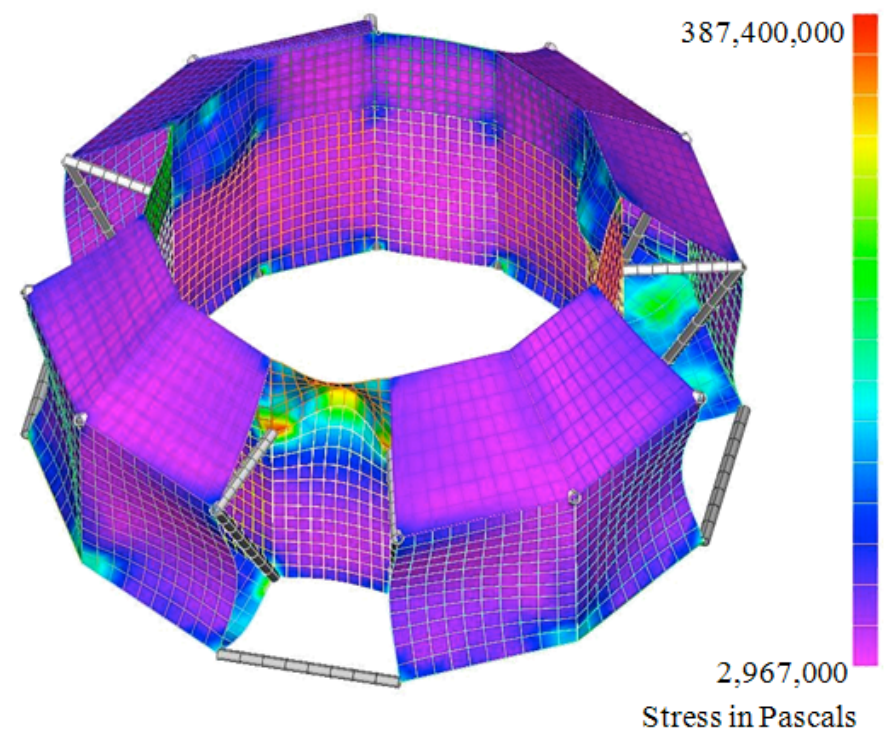

Figure 6. Spacecraft bus stresses during launch.

Table 9. Structures and mechanisms system component masses.

\begin{tabular}{|c|c|}
\hline Element & Mass (kg) \\
\hline Inner Lower Panel & 220 \\
\hline Inner Upper Panel & 102.4 \\
\hline Upper Panel & 301.6 \\
\hline Outer Panel & 269.6 \\
\hline Bottom Panel & 192.8 \\
\hline Inner Lower Tank Panel & 110 \\
\hline Inner Upper Tank Panel & 77.2 \\
\hline Tank Cross Bar & 2 \\
\hline Internal Angle Bar & 15.6 \\
\hline Internal Horiz Bar & 8.88 \\
\hline Docking System Reflectors & 5 \\
\hline Docking System Mechanical Interface & 20 \\
\hline Structures \& Mechanisms Total & 1,300 \\
\hline
\end{tabular}

\section{SERVICING}

An earlier large monolithic telescope study ${ }^{7}$ very similar to the ATLAST 8-meter study showed that designing spacecraft components to survive 20 years in space is very expensive, and designing the spacecraft such that components can be replaced during servicing missions can greatly reduce the cost of the spacecraft. Servicing also allows replacement of science instruments as well as allowing technological upgrades to spacecraft components. Given the obvious benefits that servicing has had on the Hubble Space Telescope (HST), the study team decided to design the spacecraft to make servicing a viable option. 
While human servicing of spacecraft has been demonstrated with HST, robotic servicing is in the early stages of development. Nevertheless, the study team decided to assume that by the time that ATLAST is operational, robotic servicing will be a viable option.

The general approach for servicing is to launch a servicing spacecraft aboard an EELV approximately every five years, bringing replacement spacecraft components and new science instruments to the observatory. The science instruments are designed to be inserted/removed from the rear of the instrument volume, and key spacecraft subsystem components or groups of components are configured in on-orbit replaceable units (ORUs) to allow removal from the rear of the spacecraft. The servicing spacecraft would use AR\&D technology and dock with one of several passive docking stations on the observatory. A robotic arm, either part of the servicing spacecraft or fixed to the observatory, could facilitate servicing. Figure 7 shows a notional servicing concept for the observatory, with the robotic arm removing an ORU from the spacecraft. An additional benefit that the servicing spacecraft can provide is storage of extra science instruments. Since the initial propellant load is sufficient for a 20 -year mission, on-orbit propellant transfer is not required.

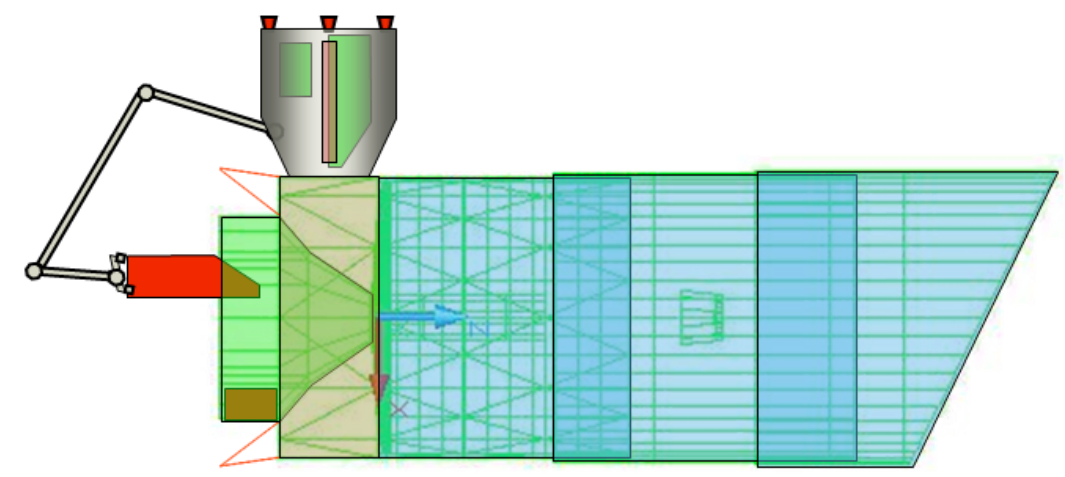

Figure 7. Notional servicing concept for the ATLAST observatory. (For clarity, the solar arrays are not shown.)

\section{ANIMATION}

The design of the solar array in both deployment and functionality are important features on the ATLAST spacecraft. These concepts required pre-visualization to provide clarity and understanding. The visualization strategy was to animate the solar array deployment and show its balancing capabilities during maneuvering. Additionally, the array boom retraction characteristics were depicted during both the main propulsion burns and science observing scenarios.

Careful consideration was made during the animation process. The goal of this work was to provide realistic solar array deployment and positioning. Considerations such as the spacecraft center of gravity and solar pressure were also captured in the animation.

The modeling, texturing, and animation work was created in Autodesk 3ds Max 2009. The rendered scenes were imported into Autodesk Combustion 2008 for background compositing and labeling of the on-screen events. Once these scenes were rendered they were edited together into one final animated video using Sony Vegas Pro 8. The final video provided clarity through visualization that aided the solar array design process. Video 1 provides a link to an animation showing the solar array deployment. Video 2 illustrates how the solar arrays are used to help offset the torque due to solar radiation pressure on the optical tube enclosure.

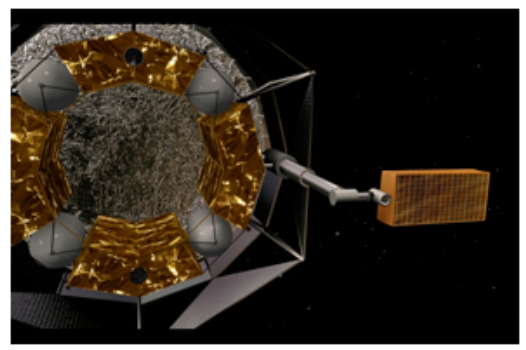

Video 1. Animation showing solar array deployment. http://dx.doi.org/doi.number.goes.here 


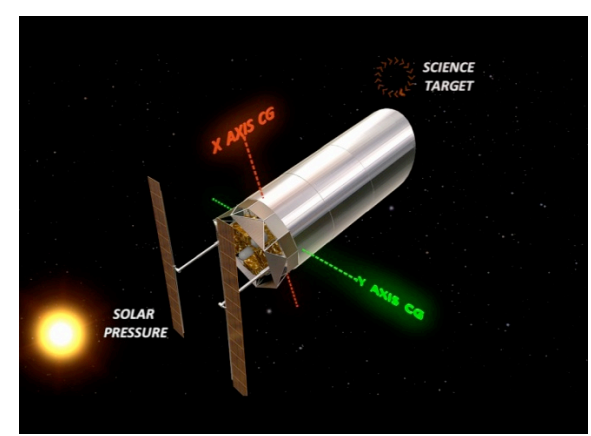

Video 2. Animation showing how the solar arrays articulate to help balance the solar radiation pressure on the optical tube enclosure: http://dx.doi.org/doi.number.goes.here

\section{CONCLUSIONS}

This study resulted in a conceptual spacecraft design capable of placing and keeping the ATLAST 8-meter observatory in a halo orbit about the second Sun-Earth Lagrange point. The Ares V heavy lift vehicle is capable of placing $65000 \mathrm{~kg}$ onto the L2 transfer trajectory with a $\mathrm{C} 3$ of $-0.7 \mathrm{~km}^{2} / \mathrm{s}^{2}$. Even though the proposed NASA budget calls for canceling the Constellation Program and thus the Ares V, the new heavy lift vehicle design study at Marshall Space Flight Center is considering launch vehicles with volume and mass capabilities comparable to the Ares V. Therefore, the possibility of launching a large monolithic observatory like the ATLAST 8-meter remains.

The ATLAST 8-meter design incorporates some technologies that need further development. While autonomous rendezvous and docking technology has been demonstrated by Orbital Express, the technology still needs to be matured. The coldplate and heatpipe connectors that must be incorporated into the science instruments and ORUs for removal and replacement during servicing may need development. Properly sized reaction wheels and control moment gyros for slewing such a large observatory do not exist, although similar systems have flown but will need to be scaled for this application. Finally, although on-orbit refueling is not necessary given the current spacecraft design and maneuver budget, this technology may be necessary if servicing requires bringing the observatory back to Earth-Moon L1 to be serviced by astronauts.

\section{REFERENCES}

[1] Postman, Marc, et. al., "Science with an 8-meter to 16-meter optical/UV space telescope", SPIE Proc. 7010, (2008).

[2] www.stsci.edu/institute/atlast

[3] Stahl, H. P., Postman, M., Arnold, W., Sr., Hopkins, R. C., Hornsby, L., Mosier, G. E., Pasquale, B. A.,"Design for an 8 meter monolithic UV/OIR space telescope," Proc. SPIE 7436 (2009).

[4] Arnold, W., Sr., and Stahl, H. P., "Structural Design Considerations for an 8-m Space Telescope," Proc. Optical Materials and Structures Technologies IV, 7425 (2009).

[5] Pedreiro, N., "Spacecraft architecture for disturbance-free payload," J. Guidance Control and Dynamics, Vol. 26, No. 5, 794-804 (2003).

[6] Hornsby, L. S., Stahl, H. P., Hopkins, R. C., "Thermal analysis of the Advanced Technology Large Aperture Space Telescope (ATLAST) 8 meter primary mirror," Proc. SPIE 7731 (2010).

[7] Hopkins, R., et al, "The Ares V L2 telescope study final report," Launch and Space Systems e-Library (LASSE), NASA Marshall Space Flight Center, Alabama, 2007. 